\title{
Quantum Dots Based Mesoporous Structured Imprinting Microspheres for the Sensitive Fluorescent Detection of Phycocyanin
}

ARTICLE in ACS APPLIED MATERIALS \& INTERFACES · APRIL 2015

Impact Factor: $5.9 \cdot$ DOI: 10.1021/acsami.5b00908

DOWNLOADS

17

5 AUTHORS, INCLUDING:

\section{Zhong Zhang}

Chinese Academy of Sciences

17 PUBLICATIONS 112 CITATIONS

SEE PROFILE

\section{Dazhong Shen}

Shandong University

51 PUBLICATIONS 378 CITATIONS

SEE PROFILE
VIEWS

61
Jinhua Li

Chinese Academy of Sciences

71 PUBLICATIONS 1,397 CITATIONS

SEE PROFILE

Lingxin Chen

Chinese Academy of Sciences

198 PUBLICATIONS 2,936 CITATIONS

SEE PROFILE 


\title{
Quantum Dots Based Mesoporous Structured Imprinting Microspheres for the Sensitive Fluorescent Detection of Phycocyanin
}

\author{
Zhong Zhang, ${ }^{\dagger} \S$ Jinhua Li, $^{\dagger}$ Xiaoyan Wang, ${ }^{\dagger, \ddagger}$ Dazhong Shen, ${ }^{\ddagger}$ and Lingxin Chen ${ }^{*} \dagger$
}

${ }^{\dagger}$ Key Laboratory of Coastal Environmental Processes and Ecological Remediation, Shandong Provincial Key Laboratory of Coastal Environmental Processes, Yantai Institute of Coastal Zone Research, Chinese Academy of Sciences, Yantai 264003, China

${ }^{\ddagger}$ Key Laboratory in Molecular and Nanomaterials Probes of the Ministry of Education of China, College of Chemistry, Chemical Engineering and Materials Science, Shandong Normal University, Jinan 250014, China

${ }^{\S}$ University of Chinese Academy of Sciences, Beijing 100049, China

\section{Supporting Information}

ABSTRACT: Phycocyanin with important physiological/environmental significance has attracted increasing attention; versatile molecularly imprinted polymers (MIPs) have been applied to diverse species, but protein imprinting is still quite difficult. Herein, using phycocyanin as template via a sol-gel process, we developed a novel fluorescent probe for specific recognition and sensitive detection of phycocyanin by quantum dots (QDs) based mesoporous structured imprinting microspheres ( $\left.\mathrm{SiO}_{2} @ \mathrm{QDs} @ m s-\mathrm{MIPs}\right)$, obeying electron-transfer-induced fluorescence quenching mechanism. When phycocyanin was present, a Meisenheimer complex would be produced between phycocyanin and primary amino groups of QDs surface, and then the photoluminescent energy of QDs would be transferred to the complex, leading to the fluorescence quenching of QDs. As a result, the fluorescent intensity of the $\mathrm{SiO}_{2} @ \mathrm{QDs} @ \mathrm{~ms}$ MIPs was significantly decreased within $8 \mathrm{~min}$, and accordingly a favorable

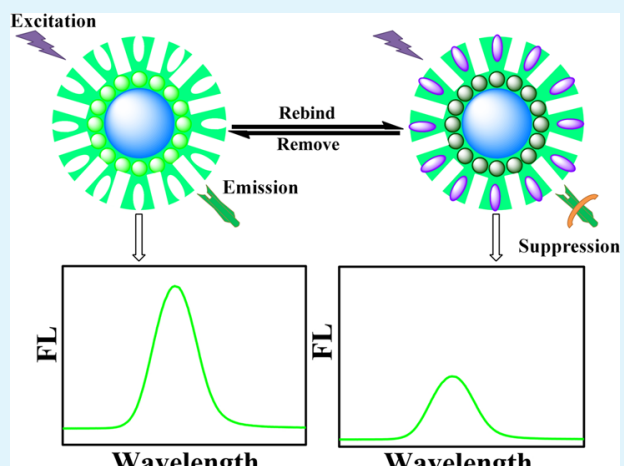

Wavelength

Wavelength linearity within $0.02-0.8 \mu \mathrm{M}$ and a high detectability of $5.9 \mathrm{nM}$ were presented. Excellent recognition specificity for phycocyanin over its analogues was displayed, with a high imprinting factor of 4.72 . Furthermore, the validated probe strategy was successfully applied to seawater and lake water sample analysis, and high recoveries in the range of $94.0-105.0 \%$ were attained at three spiking levels of phycocyanin, with precisions below 5.3\%. The study provided promising perspectives to develop fluorescent probes for convenient, rapid recognition and sensitive detection of trace proteins from complex matrices, and further pushed forward protein imprinting research.

KEYWORDS: quantum dots, fluorescent detection, molecularly imprinted polymers, mesoporous structure, phycocyanin

\section{INTRODUCTION}

The development of fluorescent labels has been driven by the demand of multiplex bioassays for simple instrumentation and processing. ${ }^{1}$ Organic dyes are often used as the conventional fluorescent labels in fluorescence immunoassay biological detection, owing to their small size, good biocompatibility, and relatively high fluorescent intensity. ${ }^{2,3}$ However, they are easily photobleached and often have narrow absorption and broad emission spectra with long tailing, which results in low detection sensitivity. ${ }^{4,5}$ Thus, semiconductor quantum dots (QDs) (e.g., CdSe, CdTe) have received increasing attention, because of their good photostability, bright photoluminescence, narrow emission and broad excitation, high quantum yields, and large Stokes shift. ${ }^{5-8}$ Based on the changes of fluorescence spectra, such QD labels have been widely used to detect various species such as ions, small organic molecules, and biomacromolecules. ${ }^{5,9,10}$ Nevertheless, selectivity is still a challenging concern of QDs based probes and sensors. ${ }^{11}$

To improve the selectivity of QDs based probes and sensors, a kind of highly selective material, molecularly imprinted polymers (MIPs), ${ }^{12}$ has been introduced as recognition units. $^{12-14}$ Recently, surface imprinting has displayed good prospects in MIPs field especially for protein imprinting compared to traditional MIPs, by virtue of its remarkable advantages such as better site accessibility, lower mass transfer resistance, more complete removal of templates, and better defined material shape. ${ }^{15,16}$ These recognition sites can mimic the binding sites of antibodies and other biological receptor molecules. Another important approach involving the detection element is chemical modification, which affords not only the desired surface binding sites but also excellent stability. ${ }^{17}$ Among various modifications of QDs for MIPs, QDs incorporation and sol-gel polymerization have received considerable attention. ${ }^{18-21}$ For example, our group has developed high-affinity dummy MIPs-capped QDs by a solgel process for the recognition and sensing of trace 2,4,6-

Received: January 30, 2015

Accepted: April 15, 2015

Published: April 15, 2015 
trinitrotoluene in soil samples matrixes. ${ }^{20}$ Wang's group has reported the formation of a MIPs based hydrophobic QDs sensor via a reverse microemulsion, and its application for the detection of ractopamine. ${ }^{21}$ Usually, these types of systems are fabricated by encapsulating QDs into silicon material so that the fluorescence of QDs are weak and the morphologies of MIPs still leave much to be desired. ${ }^{20,21}$ In order to further improve the morphology of particles, as well as to make such systems possess favorable fluorescence spectra, some support materials have been used, such as silica nanoparticles and magnetic particles. ${ }^{22-24}$

Among the support materials, silica nanoparticles are especially popular to improve the morphology of core-shell MIPs particles. The rigid, highly cross-linked structure of silica by sol-gel polymerization allows the creation of delicate recognition sites compared to more flexible organic polymers, owing to the unique properties of silica such as inertness and biocompatibility. ${ }^{25,26}$ MIPs based sensors and probes are prepared to link lots of QDs "satellites" on the surface of $\mathrm{SiO}_{2}$ nanoparticles, and the QDs can be situated at the surface or in the proximity of the surface, so the fluorescence of QDs is strong. MIPs based composite materials combine the favorable molecular recognition specificity of MIPs with additional functions of other materials such as mesoporous silica, which are more suitable in practical applications. ${ }^{24}$ Mesoporous silica materials possess large pore volume and nanosized pore wall thickness and therefore have become popular substrates in surface imprinting. ${ }^{27}$ The recognition sites of imprinted mesoporous silica materials are located near to the surface, which offers high accessibility for target molecules; this is especially important for a highly cross-linked matrix because it hinders molecules from moving freely. ${ }^{28}$ Accordingly, we have reasons to expect that developing such mesoporous structured MIPs composite systems will particularly facilitate addressing the quite difficult issue of protein imprinting.

Phycocyanin, a blue colored photosynthetic accessory pigment protein, exhibits significant biological and biomedical values. $^{29}$ Also, it has important environmental significance, because it can be used as an effective indicator of cyanobacterial blooms owing to its close relationship with cyanobacterial biomass. ${ }^{30}$ Besides the reported remote sensing methods, ${ }^{30}$ in which empirical and semiempirical models are commonly used to estimate the relationship between phycocyanin concentrations and water quality, few measurement methods have been developed. ${ }^{31,32}$ So, it is quite imperative to develop effective strategies for recognition and determination of phycocyanin.

In this work, we proposed a novel QDs based mesoporous structured imprinting microspheres probe strategy via a sol-gel polymerization for the fluorescent detection of phycocyanin based on electron-transfer-induced fluorescence quenching mechanism. $\mathrm{SiO}_{2}$ nanoparticles were used as support materials, QDs were linked on its surface, and then the mesoporous structured imprinting silica shell was deposited, to fabricate the unique mesoporous structured MIPs microspheres probe, namely, SiO $2 @ Q D s @ m s-M I P s . T h e$ microspheres possessed $\mathrm{CdTe}$ QDs as fluorescence detection units and mesoporous structured imprinting silica shell as selective recognition units, along with $\mathrm{SiO}_{2}$ nanoparticles as support cores. The probe was well characterized, and its binding capacity, response time, sensitivity, stability, and selectivity were investigated systematically. Consequently, the probe was successfully applied to the detection of trace phycocyanin in seawater and lake water samples with satisfactory results, indicating its great application potential for specific recognition and accurate quantification of proteins in complex matrices.

\section{EXPERIMENTAL SECTION}

2.1. Materials. Phycocyanin, phycoerythrin, and spirulina powder were kindly supplied by Shandong Oriental Ocean Co. (Yantai, China). Albumin from chicken egg (CEA), bovine serum albumin (BSA), lysozyme (LYZ), 2-N-morpholinoethanesulfonic acid (MES), 3-aminopropyltriethoxysilane (APTES), cetyltrimethylammonium bromide (CTAB), 3-ethylcarbodiimide hydrochloride (EDC), and $\mathrm{N}$ hydroxysuccinimide (NHS) were purchased from Sigma-Aldrich (Shanghai, China). Tellurium powder, Triton $\mathrm{X}-100$, cadmium nitrate $\left(\mathrm{Cd}\left(\mathrm{NO}_{3}\right)_{2}\right)$, and sodium borohydride $\left(\mathrm{NaBH}_{4}\right)$ were purchased from Shanghai Chemical Reagents Co. (Shanghai, China). Sodium hydroxide $(\mathrm{NaOH})$, tetraethoxysilicane (TEOS), ammonia solution $\left(\mathrm{NH}_{3} \cdot \mathrm{H}_{2} \mathrm{O}\right)$, hydrochloric acid $(\mathrm{HCl})$, thioglycollic acid (TGA), ethanol, phosphate buffered saline (PBS), and other affiliated materials were all provided by Sinopharm Chemical Reagent Co. Ltd. (Shanghai, China). All solvents, chemicals, and materials were of at least analytical pure grade and used directly without further purification unless otherwise specified. All aqueous solutions throughout the work were prepared with ultrapure water (18.2 $\mathrm{M} \Omega$ specific resistance), which was produced by a Pall Cascada laboratory water system (Millipore, Bedford, MA, USA).

2.2. Synthesis of Amino-Functionalized $\mathrm{SiO}_{2}$ Nanoparticles. Silica $\left(\mathrm{SiO}_{2}\right)$ nanoparticles were synthesized based on the Stöber method $^{33}$ with necessary modification. Briefly, $30 \mathrm{~mL}$ of ethanol and $50 \mathrm{~mL}$ of ultrapure water were mixed, followed by adding $10 \mathrm{~mL}$ of $\mathrm{NH}_{3} \cdot \mathrm{H}_{2} \mathrm{O}$, and the mixture solution was magnetically stirred under room temperature for uniformity. Then, $5 \mathrm{~mL}$ of TEOS and $20 \mathrm{~mL}$ of ethanol were added drop by drop with a constant-pressure dropping funnel, and then the resultant mixture solution was stirred for $6 \mathrm{~h}$. A 5 $\mathrm{mL}$ aliquot of APTES was then added, and the mixed solution was magnetically stirred for $12 \mathrm{~h}$ under room temperature. The obtained amino-functionalized $\mathrm{SiO}_{2}$ nanoparticles were purified by repeated centrifugation and washing with ethanol. Then the amino-functionalized $\mathrm{SiO}_{2}$ nanoparticles were dispersed in $50 \mathrm{~mL}$ of ethanol, for use.

2.3. Synthesis of Carboxylated CdTe QDs. The carboxylated CdTe QDs were synthesized according to our previous report. ${ }^{20}$ As a result, the TGA stabilized carboxylated CdTe QDs were obtained.

2.4. Preparation of $\mathrm{SiO}_{2} @ Q D s$. A $5 \mathrm{~mL}$ aliquot of aminofunctionalized $\mathrm{SiO}_{2}$ solution was dispersed into $45 \mathrm{~mL}$ of MES buffer $(0.1 \mathrm{mM})$. Meanwhile, $6 \mathrm{~mL}$ of EDC $(20 \mathrm{mg} / \mathrm{mL})$ and $6 \mathrm{~mL}$ of NHS $(10 \mathrm{mg} / \mathrm{mL})$ were successively added into $10 \mathrm{~mL}$ of carboxylated CdTe QDs solution. After the carboxyl groups were activated, the QDs solution was added drop by drop into the preceding $\mathrm{SiO}_{2}$ solution. ${ }^{22,34}$ The resultant mixture was stirred for $12 \mathrm{~h}$ in the dark at room temperature. Then the obtained $\mathrm{SiO}_{2} @ \mathrm{QDs}$ composite nanoparticles were purified by repeated centrifugation at $7000 \mathrm{rpm}$ for $5 \mathrm{~min}$, to remove the unbound QDs, and finally the obtained nanoparticles were dispersed into $50 \mathrm{~mL}$ of PBS solution $(0.01 \mathrm{M}, \mathrm{pH}$ $7.5)$

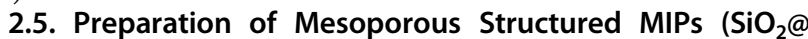
QDs@ms-MIPs).A10 mL aliquot of $\mathrm{SiO}_{2} @ Q D s$ nanoparticles solution was dispersed into $30 \mathrm{~mL}$ of ultrapure water and then was ultrasonicated for $10 \mathrm{~min}$. A $160 \mu \mathrm{L}$ aliquot of APTES and $20 \mathrm{mg}$ of phycocyanin were added and stirred for $30 \mathrm{~min}$, and then $1.6 \mathrm{~mL}$ of CTAB $(0.2 \mathrm{M})$ and $0.2 \mathrm{~mL}$ of $\mathrm{NaOH}(0.2 \mathrm{M})$ were added. After stirring for $30 \mathrm{~min}, 200 \mu \mathrm{L}$ of TEOS and $200 \mu \mathrm{L}$ of $\mathrm{NH}_{3} \cdot \mathrm{H}_{2} \mathrm{O}$ were added to the mixture and kept stirring for $12 \mathrm{~h}$ in the dark. The products were washed three times with a mixture solvent of ethanol/ $0.1 \mathrm{M} \mathrm{HCl}(8: 2, \mathrm{v} / \mathrm{v})$ to remove phycocyanin and $\mathrm{CTAB}$ and then were dried in a vacuum oven at $40{ }^{\circ} \mathrm{C}$. Finally, the mesoporous structured products were attained, namely, SiO $2 @ Q D s @ m s-M I P s$, and for simplicity, MIPs were also used to indicate the products. On the other hand, mesoporous structured nonimprinted polymers, i.e., $\mathrm{SiO}_{2} @ \mathrm{QDs} @ m s-\mathrm{NIPs}$ (NIPs, for simplicity), were also prepared by the same procedure without the addition of template phycocyanin. 


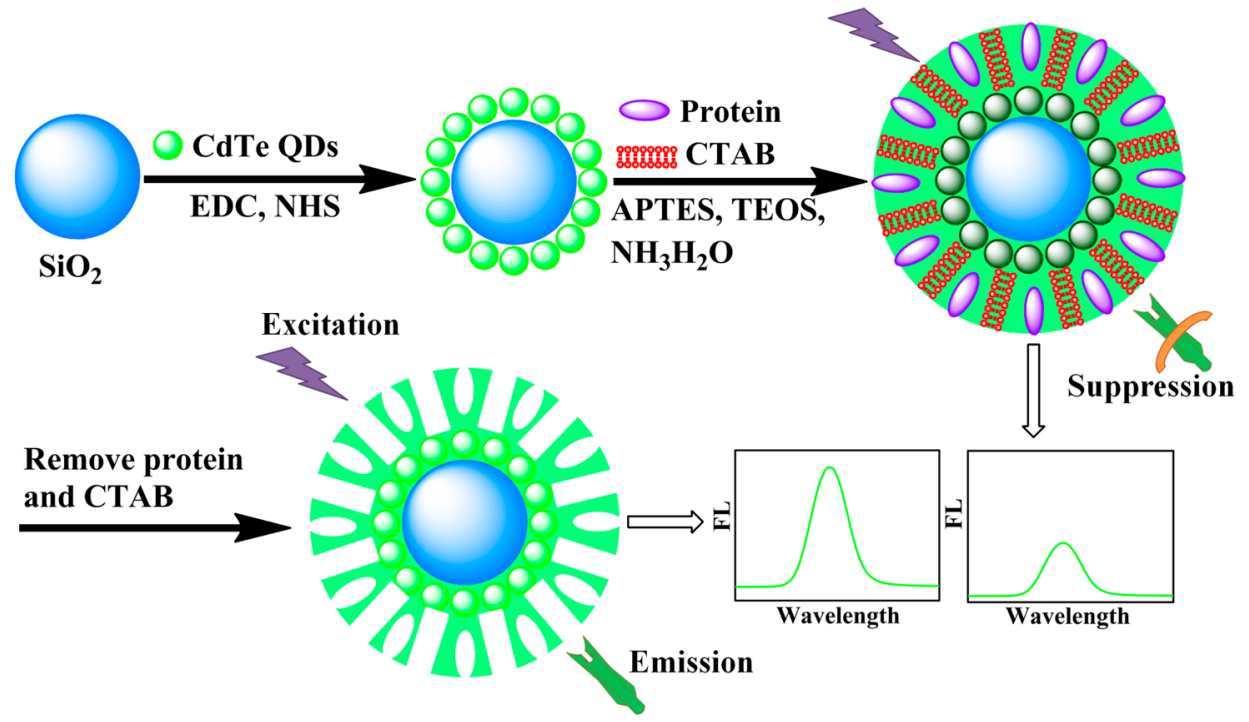

Figure 1. Schematic illustrations for the preparation process of $\mathrm{SiO}_{2} @ \mathrm{QDs} @ m s-M I P s$.

Moreover, as a control, $\mathrm{SiO}_{2} @ \mathrm{QDs} @ \mathrm{MIPs}$ were also prepared in the same way as $\mathrm{SiO}_{2} @ \mathrm{QDs} @ m s-\mathrm{MIPs}$ without adding CTAB.

2.6. Characterization. The morphological evaluation was performed by scanning electron microscope (SEM, JSM $5600 \mathrm{LV}$, operating at $20 \mathrm{kV}$ ), transmission electron microscope (TEM, JEM1230 , operating at $100 \mathrm{kV}$ ), and high resolution TEM (HRTEM, JEM$2010 \mathrm{HR}$, operating at $80 \mathrm{kV}) . \mathrm{N}_{2}$ adsorption-desorption isotherms and structure parameters were determined via Brunauer-EmmettTeller (BET) analysis by Full-Automatic Specific Surface Instruments (3H-2000BET-A, Beishide Instruments). Infrared spectra were obtained from Fourier transform infrared spectrometer (Nicolet iS10, Thermo Scientific) to examine the preparation process. Fluorescence spectra were recorded using a spectrofluometer (Fluoromax-4, HORIBA): excitation light was set at $590 \mathrm{~nm}$, and the emission spectra were measured from 605 to $700 \mathrm{~nm}$ for phycocyanin, and the silt widths of excitation and emission were set at 5 and $5 \mathrm{~nm}$, respectively; ${ }^{31}$ excitation light was set at $397 \mathrm{~nm}$ and the emission spectra were recorded from 450 to $600 \mathrm{~nm}$ for QDs. A fluorescence microscope (BX61, Olympus) was used to observe the fluorescence microscopy image on a glass slide equipped with a CCD camera. UV/vis absorption spectra were recorded to test the binding properties of polymers on a spectrophotometer (NanoDrop 2000/ 2000C, Thermo Scientific) at the wavelength of $280 \mathrm{~nm}$. Sodium dodecyl sulfate polyacrylamide gel electrophoresis (SDS-PAGE) experiment was conducted with 5\% stacking gel and $15 \%$ running gel.

2.7. Analysis of Water Samples. Water samples were utilized to examine the practical applicability of the $\mathrm{SiO}_{2} @ \mathrm{QDs} @ m s-M I P s$ for the phycocyanin detection. Surface seawater samples from the Fisherman's Wharf of the Yellow Sea located in the coastal zone area of Yantai City were gathered into a Teflon bottle. Lake water samples from an artificial lake located in Laishan District of Yantai City were collected into a Teflon bottle. And the water samples were all filtrated through $0.45 \mu \mathrm{m}$ PTFE syringe filters (Phenomenex, Los Angeles, CA, USA) to remove the suspended particles before use.

A6 mg amount of $\mathrm{SiO}_{2} @ \mathrm{QDs} @ \mathrm{~ms}-\mathrm{MIPs}$ (or $\mathrm{SiO}_{2} @ \mathrm{QDs} @ \mathrm{~ms}$ NIPs) was dispersed into $2 \mathrm{~mL}$ of 100 -fold diluted seawater samples spiked with the mixture of phycocyanin, CEA, and BSA individual at 1 $\mathrm{mg} / \mathrm{mL}$, and then the mixture was incubated for $12 \mathrm{~h}$ at room temperature under gently shaking. Finally, $10 \mu \mathrm{L}$ of the supernatant solutions of each sample was collected and analyzed by SDS-PAGE.

The spiked seawater and lake water samples were used to validate the accuracy and practicality of the $\mathrm{SiO}_{2} @ \mathrm{QDs} @ m s-\mathrm{MIP}$ probe. Because no phycocyanin was detectable in the collected water samples, recovery experiments were carried out with phycocyanin for the spiked test. A 5 mg amount of $\mathrm{SiO}_{2} @ \mathrm{QDs} @ m s-M I P s$ was first dispersed into $10 \mathrm{~mL}$ of ultrapure water, and then $0.1 \mathrm{~mL}$ of the solution was added into $2 \mathrm{~mL}$ of a 100 -fold dilution of seawater sample or $2 \mathrm{~mL}$ of lake water sample, spiked with phycocyanin at three concentration levels of $0.1,0.2$, and $0.5 \mu \mathrm{mol} / \mathrm{L}$. Then the fluorescence spectra were recorded. All of the tests were carried out in triplicate, and the average values from triplicate independent results were utilized for the following discussion.

\section{RESULTS AND DISCUSSION}

3.1. Preparation of $\mathrm{SiO}_{2} @ \mathrm{QDs} @ m s-M I P s$. Figure 1 schematically illustrates the preparation process of $\mathrm{SiO}_{2} @$ $\mathrm{QDs} @ \mathrm{~ms}-\mathrm{MIPs}$. As seen, $\mathrm{SiO}_{2}$ nanoparticles were used as core support materials, and lots of CdTe QDs "satellites" were linked on the surface of the amino-functionalized $\mathrm{SiO}_{2}$ core by virtue of amide bonding. Then, functional monomer (APTES), template molecule (phycocyanin), cross-linker (TEOS), catalyst $\left(\mathrm{NH}_{3} \cdot \mathrm{H}_{2} \mathrm{O}\right)$, and surfactant $(\mathrm{CTAB})$ were added to form the imprinted silica shell on the surface of $\mathrm{SiO}_{2} @ \mathrm{QDs}$. After removing the embedded template protein phycocyanin and surfactant CTAB, SiO ${ }_{2} @ Q D s @ m s-M I P s$ with specific imprinted cavities and mesoporous structures were obtained. The ms-MIPs silica shell layer could not only protect the fluorescence of QDs and effectively decrease QDs toxicity but also facilitate high accessibility into binding sites and rapid mass transfer of template molecules. In the presence of phycocyanin, a Meisenheimer complex would be produced between phycocyanin and primary amino groups on the surface of the QDs, and then the photoluminescent energy of QDs would be transferred to the complex and result in QDs fluorescence quenching. So, the phycocyanin could be detected fluorescently by the $\mathrm{SiO}_{2} @ \mathrm{QDs} @ \mathrm{~ms}-\mathrm{MIPs}$.

3.2. Characterization of $\mathrm{SiO}_{2} @ \mathrm{QDs} @ m s-M I P s$. The morphological structures of $\mathrm{SiO}_{2} @ \mathrm{QDs}$ and $\mathrm{SiO}_{2} @ \mathrm{QDs} @ \mathrm{~ms}$ MIPs were characterized by SEM and TEM. It was observed from Figure 2A,B that $\mathrm{SiO}_{2}$ nanoparticles had good dispersion and the average diameter was about $70-90 \mathrm{~nm}$; the addition of more water allowed formation of a much smaller size than that prepared by the original Stöber method $(220-400 \mathrm{~nm}){ }^{33}$ The monodispersed $\mathrm{SiO}_{2}$ nanoparticles were used as core support materials. As seen, CdTe QDs satellites were linked on the surface of amino-functionalized $\mathrm{SiO}_{2}$, and the QDs size was about $10 \mathrm{~nm}$, revealing the mosaic structures of CdTe QDcoated $\mathrm{SiO}_{2}$ particles were successfully prepared (Figure 2A,B). 

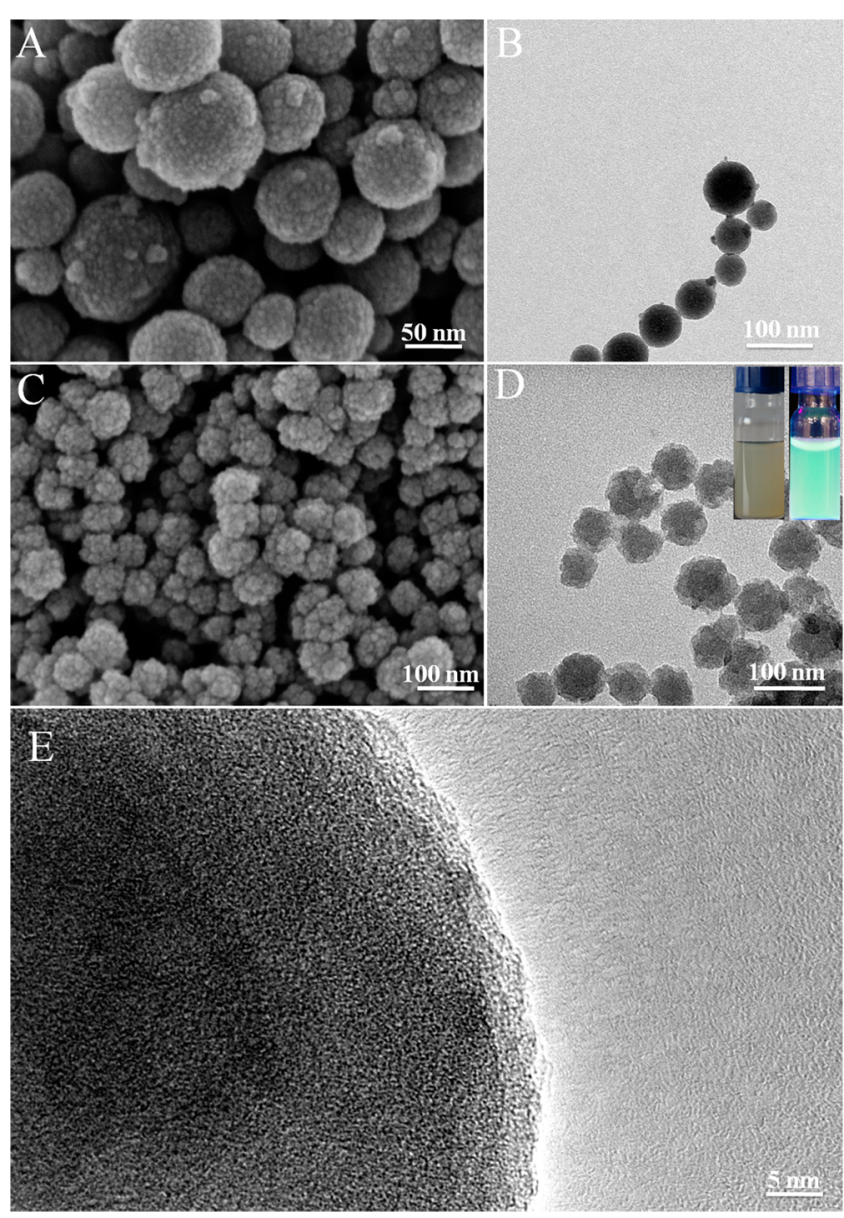

Figure 2. (A, B) SEM and TEM images of $\mathrm{SiO}_{2} @ Q D s,(C, D)$ SEM and TEM images of $\mathrm{SiO}_{2} @ Q D s @ m s-M I P s$, and (E) HRTEM image of $\mathrm{SiO}_{2} @ \mathrm{QDs} @ m s-\mathrm{MIPs}$. (Inset of D: photographs of $\mathrm{SiO}_{2} @ \mathrm{QDs} @$ ms-MIPs solution under sunlight (left) and ultraviolet lamps (right)).
As seen from Figure 2C,D, MIPs exhibited core-shell structures and the MIP layer could be estimated to be about $5 \mathrm{~nm}$ in thickness on the surface of $\mathrm{SiO}_{2}$ nanoparticles. Noticeably, the MIP particles displayed a highly rough outside polymeric surface, indicating the presence of a large number of exposed complementary imprinted cavities on the surface. The rough surfaces of $\mathrm{SiO}_{2} @ \mathrm{QDs} @ m s-\mathrm{MIPs}$ and those worm-like channels suggested mesoporous structures existed in the silica shell, as shown in Figure 2E. In addition, the mesoporous structure of the $\mathrm{SiO}_{2} @ \mathrm{QDs} @ \mathrm{~ms}-\mathrm{MIPs}$ was further confirmed by BET analysis. The $\mathrm{SiO}_{2} @ \mathrm{QDs} @$ ms-MIPs specific surface area attained was $187.1 \mathrm{~m}^{2} / \mathrm{g}$, higher than that of the corresponding SiO $@ @ Q D s @ M I P s\left(125.9 \mathrm{~m}^{2} / \mathrm{g}\right)$, suggesting the presence of mesoporous structure. The $\mathrm{N}_{2}$ sorption isotherm of the $\mathrm{SiO}_{2} @ \mathrm{QDs} @ m s-M I P s$ exhibited a rapid increase in the adsorption branch at a relative pressure of 0.4-0.6, which clearly indicated considerable numbers of uniform mesoporouses were present in the $\mathrm{SiO}_{2} @ \mathrm{QDs} @ m s-$ MIPs. And the uniform mesoporous had an average diameter of $4.49 \mathrm{~nm}$, obtained from the Barrett-Joyner-Halenda (BJH) pore size distribution curve (the inset of Figure S1, Supporting Information). This structure made mass transfer resistance lower, site accessibility higher, and material shape better defined for special recognition toward the template phycocyanin, which would provide a faster moving rate and higher binding capacity. As proved in the inset of Figure 2D, under the irradiation of an ultraviolet lamp, MIPs had relatively strong green fluorescence. The result proved that the hybrid structure was successfully synthesized, which possessed the CdTe QDs as sensitive fluorescence signal units and the thin mesoporous structured imprinted silica shell layer as selective recognition units.

Supporting Information (SI) Figure S2 shows the FT-IR spectra of $\mathrm{SiO}_{2}(\mathrm{a}), \mathrm{SiO}_{2} @$ QDs (b), and $\mathrm{SiO}_{2} @$ QDs-ms-MIPs (c) particles. As seen, the characteristic peak around 466 and $794 \mathrm{~cm}^{-1}$ could be ascribed to $\mathrm{Si}-\mathrm{O}$ stretching vibration, and the wide and strong absorption band around $1091 \mathrm{~cm}^{-1}$ could
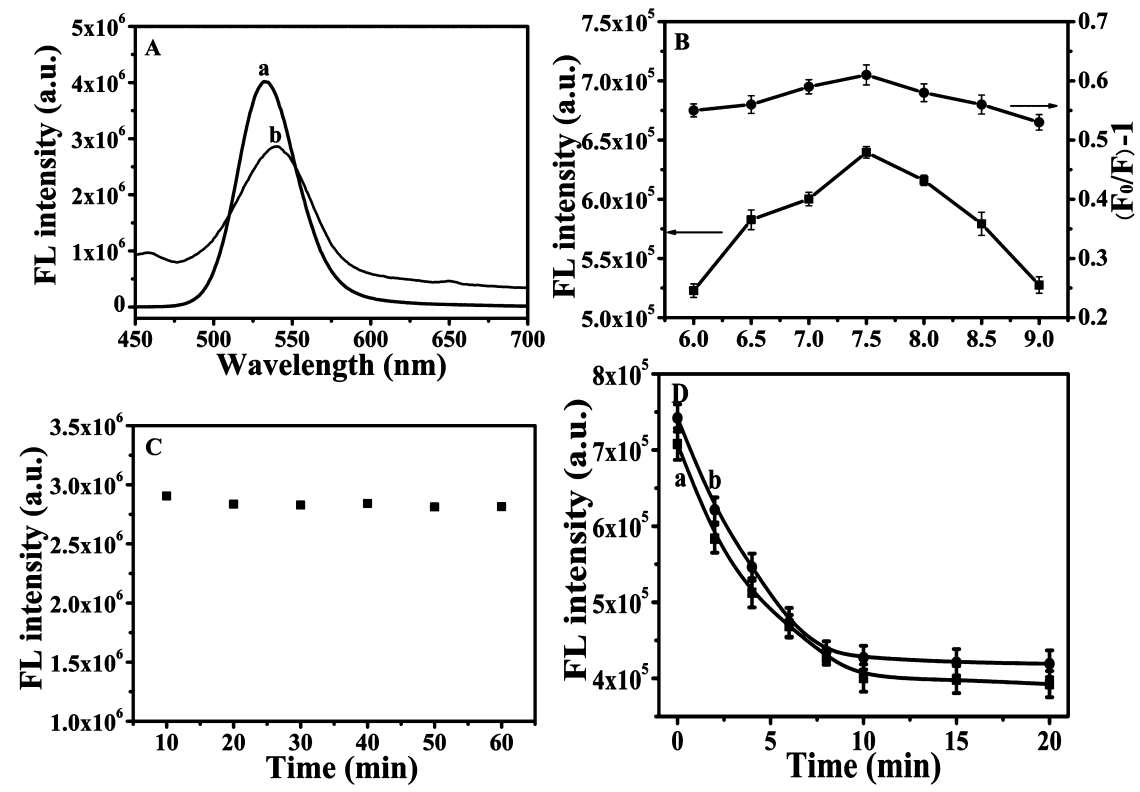

Figure 3. (A) Fluorescence spectra of QDs (a) and $\mathrm{SiO}_{2} @ \mathrm{QDs} @ m s-\mathrm{MIPs}(\mathrm{b})$. (B) Effect of pH on fluorescence intensity changes of $\mathrm{SiO}{ }_{2} @ \mathrm{QDs} @$ ms-MIPs (left) and fluorescence quenching changes of $\mathrm{SiO}_{2} @ \mathrm{QDs} @ \mathrm{~ms}-\mathrm{MIPs}$ (right) caused by $0.1 \mathrm{mg} / \mathrm{L}$ phycocyanin. (C) Fluorescence intensity change of $\mathrm{SiO}_{2} @ \mathrm{QDs} @ \mathrm{~ms}-\mathrm{MIPs}$ within60 min. (D) Fluorescence response time of $\mathrm{SiO}_{2} @ \mathrm{QDs} @ \mathrm{MIPs}(\mathrm{a})$ and $\mathrm{SiO}_{2} @ \mathrm{QDs} @ \mathrm{~ms} \mathrm{MIPs}(\mathrm{b})$ for 0.1 mg/L phycocyanin. Experimental conditions: SiO ${ }_{2} @ Q D s @ m s-M I P s$ or $\mathrm{SiO}_{2} @ Q D s @ M I P s, 25$ mg/L; excited light, 397 nm. 
be attributed to stretching vibrations of $\mathrm{Si}-\mathrm{O}-\mathrm{Si}$, indicating the occurrence of $\mathrm{SiO}_{2}$ matrices in the three materials. The stretching vibration of $\mathrm{N}-\mathrm{H}$ at 1631 and $3436 \mathrm{~cm}^{-1}$ proved the amino group was modified onto the surface of $\mathrm{SiO}_{2}$ nanoparticles (SI Figure S2a). As shown in SI Figure S2b, a new absorption peak at about $1411 \mathrm{~cm}^{-1}$ could be assigned to the $\mathrm{C}-\mathrm{N}$ stretching vibration of the acylamino group, which proved the successful grafting of $\mathrm{QDs}$ on $\mathrm{SiO}_{2}$. No other characteristic peaks appeared for the $\mathrm{SiO}_{2} @$ QDs-ms-MIPs, while the characteristic absorption peaks for $\mathrm{SiO}_{2}$ and the acylamino group became weakened significantly, as displayed in SI Figure S2c. The results indicated that a thin imprinted shell layer was successfully modified on the surface of the $\mathrm{SiO}_{2} @$ QDs particles.

SI Figure S3 shows the thermogravimetry (TG) and derivative thermogravimetry (DTG) curves of $\mathrm{SiO}_{2} @ \mathrm{QDs} @$ ms-MIPs. With the increase of temperature from 40 to $100{ }^{\circ} \mathrm{C}$, the weight loss may be caused by the volatile loss of absorbed water. The loss amounts were $2.18 \%$. In the range of $100-320$ ${ }^{\circ} \mathrm{C}$, the weight loss rate was very slow, suggesting the MIPs were very stable below $320{ }^{\circ} \mathrm{C}$. In the range of $320-580{ }^{\circ} \mathrm{C}$, remarkable loss amounts were shown with $6.59 \%$, and the weight loss was highly likely to be caused by the decomposition of organic material in the MIPs. The peak temperature of MIPs was found at $420^{\circ} \mathrm{C}$. It should be noted that the curve showed a certain increase within $600-800{ }^{\circ} \mathrm{C}$, which is very likely owing to the presence of a $\mathrm{SiO}_{2}$ core and a large number of mesoporouses in the MIPs. In view of the conventional temperature, it could be concluded that the prepared MIPs were fully demonstrated to occupy good thermal stability at the temperature lower than $320{ }^{\circ} \mathrm{C}$. These results could also be clearly observed from the DTG curve.

3.3. Binding Performances of the $\mathrm{SiO}_{2} @ \mathrm{QDs} @ m s-M I P s$ for Phycocyanin. In order to investigate the binding performances of the $\mathrm{SiO}_{2} @ \mathrm{QDs} @ m s-\mathrm{MIPs}$ and $\mathrm{SiO}_{2} @ \mathrm{QDs} @$ ms-NIPs, the adsorption capacity analysis was carried out using $0.5 \mathrm{mg} / \mathrm{mL}$ of phycocyanin. As shown in SI Figure S4, the adsorption capacity of the MIPs was $9.95 \mathrm{mg} / \mathrm{g}$, much larger than that of the NIPs $(2.96 \mathrm{mg} / \mathrm{g})$. This indicated that a large number of specific binding sites were produced during the MIPs preparation process after removing the template molecules. So, the MIPs could be employed as an ideal adsorption material for selective recognition and enrichment of phycocyanin.

3.4. Fluorescence Properties of the $\mathrm{SiO}_{2} @ \mathrm{QDs} @ m s-$ MIPs. In this work, after MIP shell encapsulation, the QDs were buried under the thin mesoporous structured imprinted silica layer. A key question was whether the encapsulated QDs would retain their fluorescence properties. As shown in Figure 3A, the $\mathrm{SiO}_{2} @ \mathrm{QDs} @ m s-M I P s$ shared fluorescence spectra similar to those of QDs (curve a), despite the fluorescence intensity decreasing a little, and the emission peak showed a slight red shift (curve b). This observation is consistent with that reported; ${ }^{20}$ that is, the electric field, which was generated by a single charge close to the QDs surface, was sufficiently large to cause fluorescence quenching and red shift. The red shift could be attributed to the formation of a hybrid structure: the resultant hybrid $\mathrm{SiO}_{2} @$ QDs@ms-MIPs could increase the effective size of the QDs and reduce the quantum size effect, which would cause a red shift of the photoluminescence maximum. ${ }^{20}$ The existent amide bond reduced the surface charge of QDs, and thereby led to the decrease of both the orientation polarization rate around QDs and the Stokes shift of the QDs. Hence, it could be deduced that the SiO $\mathrm{O}_{2} @ \mathrm{QDs} @$ ms-MIPs possessed good fluorescence properties.

The solution acidity played an important part in the fluorescence property because it had remarkable influences on the three-dimensional structure, the charge of phycocyanin, and the fluorescence intensity of $\mathrm{SiO}_{2} @ \mathrm{QDs} @ m s-M I P s$. And it hence affected the rebinding of phycocyanin at different $\mathrm{pH}$ values. The isoelectric point (PI) value of phycocyanin was determined as 4.3. Figure $3 \mathrm{~B}$ shows the effects of $\mathrm{pH}$ on the fluorescence intensity change of MIPs (left), and on fluorescence quenching amount change of MIPs in the presence of phycocyanin (right). The fluorescence intensity was low at $\mathrm{pH}$ less than 7.0. The fluorescence intensity increased along with an increase in $\mathrm{pH}$ from 6 to 7.5 , and it approached a maximum at $\mathrm{pH}$ 7.5. The quenching changes of MIPs caused by phycocyanin shared a similar trend to the fluorescence intensity changes. When $\mathrm{pH}$ was higher than 7.5 , the imprinting silica layer could be ionized and thereby could decrease the interaction between the template phycocyanin and the imprinted cavities. And phycocyanin may be slightly denatured at higher or lower $\mathrm{pH}$ values. In view of possible applications of the $\mathrm{SiO}_{2} @ \mathrm{QDs} @ \mathrm{~ms}-\mathrm{MIPs}$ in seawater, pH 7.5 was selected for the following work.

Under optimized conditions, the fluorescence stability was examined by repeatedly detecting the fluorescence intensity every $10 \mathrm{~min}$ at maximum emission peak. The result shown in Figure 3C revealed a stable emission of $\mathrm{SiO}_{2} @ \mathrm{QDs} @ m s-\mathrm{MIPs}$ within $60 \mathrm{~min}$. In addition, because of the highly cross-linked polymeric characteristic, the fluorescence probe displayed good physical stability and chemical inertness. The fluorescence probe could be easily recovered, by eluting with methanolacetate solution to remove rebound phycocyanin and rinsing with a neutral PBS to restore to the neutral blank status. So, the fluorescence probe proved excellently reproducible owing to the recoverability of five cycles with relative standard deviation (RSD) of 5.2\%. Moreover, about the storage stability, the repeated detection of the fluorescence intensity was recorded every week and the intensity retained $94.7 \%$ of its initial intensity, which implied that the fluorescence probe had acceptable storage stability. Furthermore, the $\mathrm{SiO}_{2} @ \mathrm{QD}$ s@msMIPs probe still provided almost consistent detection results after it was stored at $4{ }^{\circ} \mathrm{C}$ in a refrigerator for 2 months. Additionally, the maintained fluorescence intensity within 60 min and 2 months showed the crucial role of the MIP silica shell which may well protect the QDs.

The response time of the fluorescence probe was also tested in order to assess the accessibility to binding sites. As seen from Figure 3D, within 4-8 min, the fluorescence intensity decrease of $\mathrm{SiO}_{2} @ \mathrm{QDs} @ m s-M I P s$ was faster (curve b), after which the curve became flat (curve b), while the $\mathrm{SiO}_{2} @ \mathrm{QDs} @ M I P s$ required $10 \mathrm{~min}$ (curve a) to reach equilibrium. The presence of mesoporous structure resulted in a shorter response time of the ms-MIPs. The generation of recognition sites between the pores of the mesoporous silica offered high accessibility of template molecules. Therefore, the ms-MIPs probe had a rapid response speed for phycocyanin, and $8 \mathrm{~min}$ was chosen as the response time for further experiments.

3.5. Possible Detection Mechanism of the $\mathrm{SiO}_{2} @ Q D s @$ ms-MIPs. The recognition and detection process for phycocyanin by the prepared $\mathrm{SiO}_{2} @ \mathrm{QDs} @ m s-\mathrm{MIPs}$ was schematically shown in Figure 1. As seen, the fluorescence quenching could be ascribed to the electron transfer between APTES and phycocyanin. The electron transfer from the 
carboxyl group to the amino groups resulted in the formation of a complex between phycocyanin and the primary amino groups. This interaction can be verified by the fluorescence spectra (SI Figure S5). When adding APTES into a phycocyanin solution, the fluorescence intensity of phycocyanin gradually decreased. This proved strong hydrogen bond forces to be present between phycocyanin and APTES. The absorption spectrum of the resultant Meisenheimer complex was found to have an excellent overlap with the emission spectrum of CdTe QDs, as shown in Figure 4A, which could very possibly result in energy transfer. Moreover, by comparing the fluorescent intensities of $\mathrm{SiO}_{2} @ \mathrm{QDs} @ m s-N I P s$ and template phycocyanin removed/
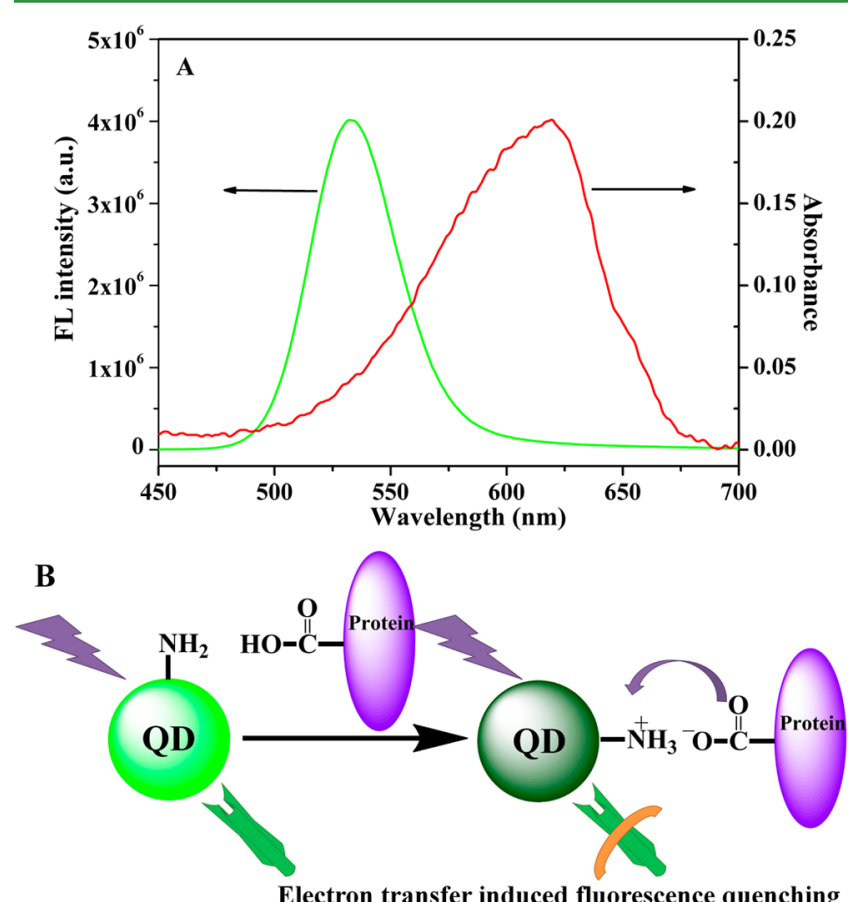

\section{Conduction band}

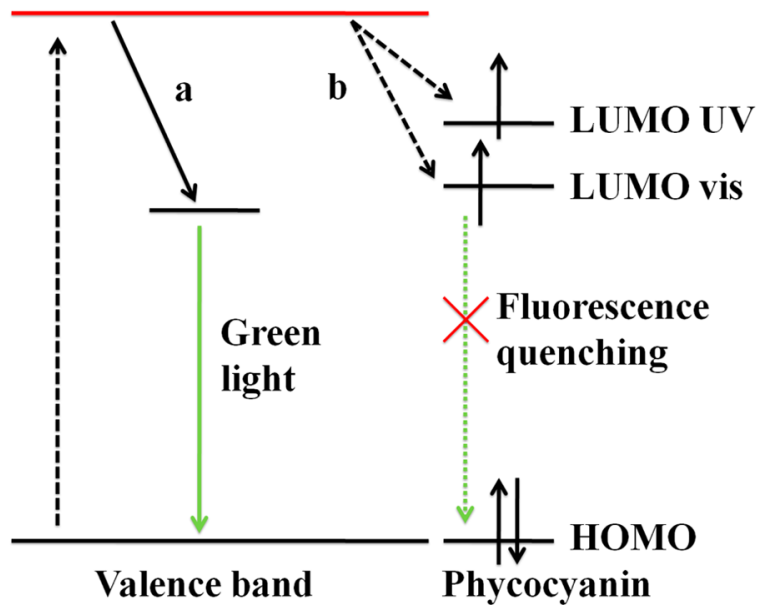

Figure 4. (A) Fluorescence emission spectra of CdTe QDs (green line) and absorption spectra of the complex of APTES with phycocyanin (red line). (B) Schematic for the QDs fluorescence quenching mechanism on the basis of the electron-transfer-induced energy transfer. (C) Schematic for the fluorescence quenching mechanism according to molecular orbital theory in the absence (a) and presence $(b)$ of phycocyanin. rebound $\mathrm{SiO}_{2} @ \mathrm{QDs} @ m s-\mathrm{MIPs}$, as shown in SI Figure S6, the significantly decreased fluorescence intensity of $\mathrm{SiO}_{2} @ \mathrm{QDs} @$ ms-MIPs with phycocyanin (green line) suggested phycocyanin was rebound by the MIPs and caused significant fluorescence quenching. Also, the fluorescent intensity of MIPs (red line) was much larger than that of NIPs (blue line), which indicated specific recognition sites with predetermined selectivity for phycocyanin were formed in the MIPs; however, the NIPs had no imprinting cavities. These results revealed energy transfer from CdTe QDs to the complex occurred and led to the QDs fluorescence quenching.

Meanwhile, the quenching mechanism could be more clearly explained, as depicted in Figure 4B,C. As seen from Figure 4B, in the presence of phycocyanin, the Meisenheimer complex could be formed between phycocyanin and the primary amino groups of APTES on the surface of the QDs. Then the energy of QDs would be transferred to the complex, resulting in the QDs fluorescence quenching. Thus, phycocyanin could be detected fluorescently. Furthermore, molecular orbital theory was adopted to interpret the quenching mechanism, schematically displayed in Figure 4C. As seen, the electron of QDs could accept the UV energy and then it would be excited from the ground state (valence band) to the conduction band; after that, the excited electron could return to the ground state. In the returning process, QDs could emit green fluorescence (Figure 4C(a)). On the other hand, with the existence of phycocyanin, a hydrogen bond could be formed between phycocyanin and the primary amino groups on the surface of the QDs. The strong interaction force would lead to electron transfer between QDs and phycocyanin. The UV and visible absorption peaks of phycocyanin are at about 227,280 , and $590 \mathrm{~nm}$, very close to the conduction band of CdTe QDs, so the excited electron could directly jump into the LUMO level layer of the Meisenheimer complex. The energy level of the complex was higher than that of CdTe QDs, so the excited electrons of QDs could return back to the ground state without emitting fluorescence, which would lead to the fluorescence quenching of QDs (Figure 4C(b)). Therefore, phycocyanin could be detected fluorescently in this way.

3.6. Sensitivity and Selectivity of the Probe. The ability of the developed $\mathrm{SiO}_{2} @$ QDs@ms-MIPs fluorescent probe for quantitative determination of phycocyanin was further evaluated. Under the optimized conditions, the fluorescence spectra of the probe to different concentrations of phycocyanin were recorded for sensitivity investigation. As shown in Figure $5 \mathrm{~A}$ (upper), the fluorescence intensities decreased distinctly with the increase of phycocyanin concentrations; an excellent linearity was presented ranging from 0.02 to $0.8 \mu \mathrm{M}$ with a correlation coefficient of 0.997 , and the precisions (RSD) for the three replicate detection of phycocyanin were within $3.2 \%$ (Figure 5A, inset). Accordingly, fluorescence changes were displayed with the increase of phycocyanin concentrations, and the strongest green fluorescence occurred when no phycocyanin was added, as seen in the bottom panel of Figure 5A. And a favorable limit of detection (LOD, $S / N=3$ ) of $5.9 \mathrm{nM}$ was attained, which meets with the requirement of trace analysis. In contrast, the decrease of fluorescence intensity of corresponding NIPs was not obvious at the same concentration of phycocyanin, as seen in Figure 5B. A comparison of Figure 5A,B clearly showed the decrease of fluorescence intensity of the MIPs was much larger than that of NIPs, indicating that the MIPs could greatly enhance the quenching efficiency and thereby enlarge the spectral sensitivity to phycocyanin owing to 

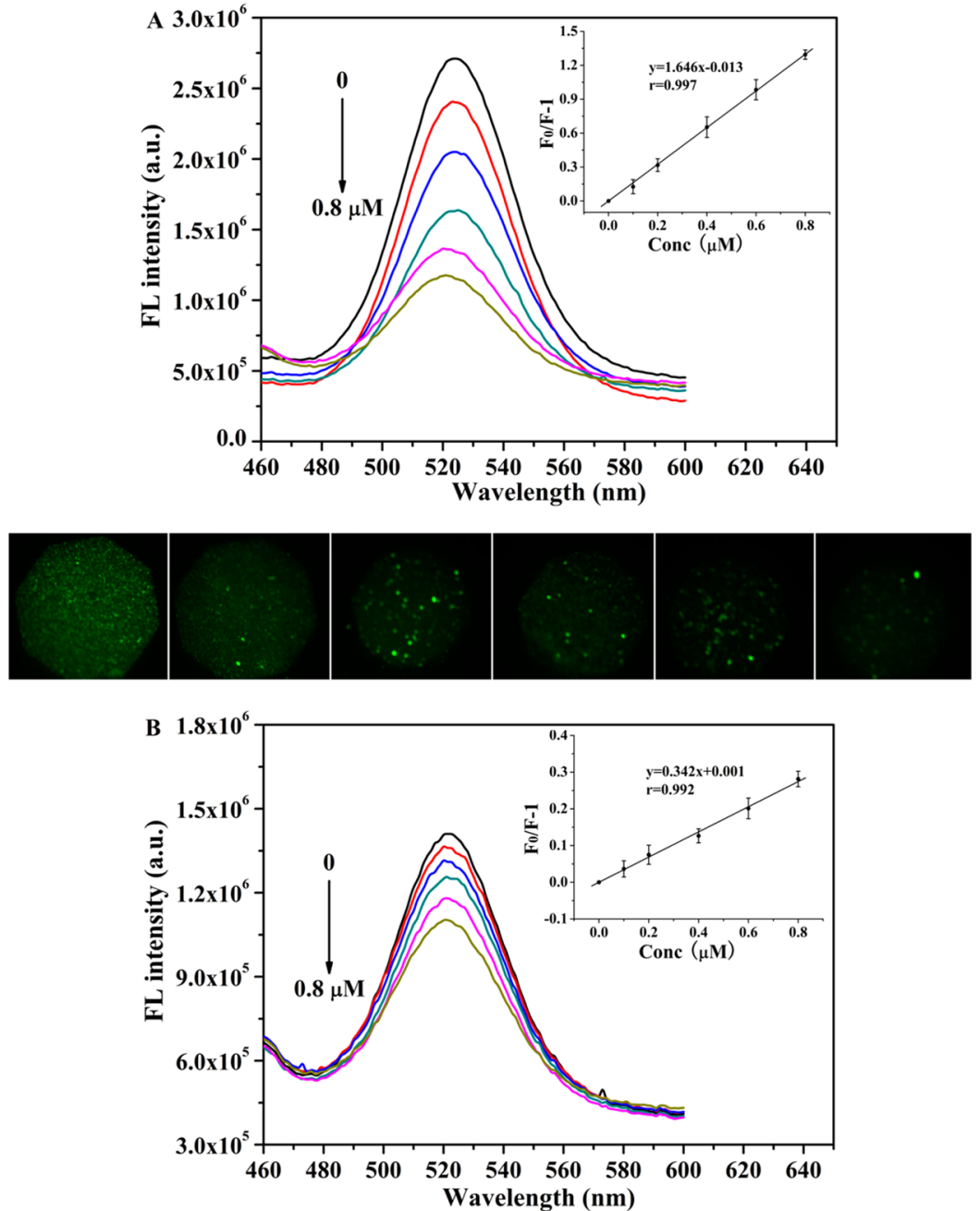

Figure 5. (A) Fluorescence emission spectra of $\mathrm{SiO}_{2} @ \mathrm{QDs} @ m s-M I P s$ with addition of the indicated concentrations of phycocyanin, respectively $(0-0.8 \mu \mathrm{M})$ (top), and corresponding fluorescence microscope images of $\mathrm{SiO}_{2} @ \mathrm{QDs} @ \mathrm{~ms}-\mathrm{MIPs}$ with addition of the indicated concentrations of phycocyanin (from left to right, concentrations, $0-0.8 \mu \mathrm{M}$ ) (bottom). (B) Fluorescence emission spectra of $\mathrm{SiO}_{2} @$ QDs@ms-NIPs with addition of the indicated concentrations of phycocyanin $(0-0.8 \mu \mathrm{M})$. The inset graphs show the Stern-Volmer plots for $\mathrm{SiO}_{2} @ \mathrm{QDs} @ \mathrm{~ms}-\mathrm{MIPs}(\mathrm{A})$ and $\mathrm{SiO} \mathrm{O}_{2} @$ QDs@ms-NIPs (B). Experimental conditions: MIPs or NIPs, $25 \mathrm{mg} / \mathrm{L}$; excited light, $397 \mathrm{~nm}$.

the formation of specific recognition sites with predetermined selectivity toward phycocyanin. Thus, the MIPs based probe could sensitively and accurately detect phycocyanin, demonstrating its feasibility for monitoring cyanobacterial bloom.

In this system, the fluorescence quenching followed the Stern-Volmer equation ${ }^{20,22,35}$

$$
F_{0} / F=1+K_{\mathrm{sv}} C_{\mathrm{q}}
$$

where $F_{0}$ and $F$ are the fluorescent intensities in the absence and presence of quencher, respectively, $K_{\mathrm{SV}}$ is the quenching constant for the quencher, and $C_{\mathrm{q}}$ is the concentration of the quencher. Herein, the ratio of $K_{\mathrm{sv}, \mathrm{MIP}}$ to $K_{\mathrm{sv}, \mathrm{NIP}}$ was defined as the imprinting factor, and $\left(F_{0} / F\right)-1$ was defined as the quenching amount. As observed in Figure 5A,B, MIPs and NIPs presented different Stern-Volmer plot relationships, and the decrease of fluorescence intensity of MIP-coated QDs was much larger than that of the NIP-coated QDs at the same phycocyanin concentration. And, a high imprinting factor could be obtained of 4.72 .

Furthermore, the selectivity of the MIPs probe was demonstrated by binding test, using phycoerythrin, spirulina powder, BSA, CEA, and LYZ. As seen from Figure 6, the

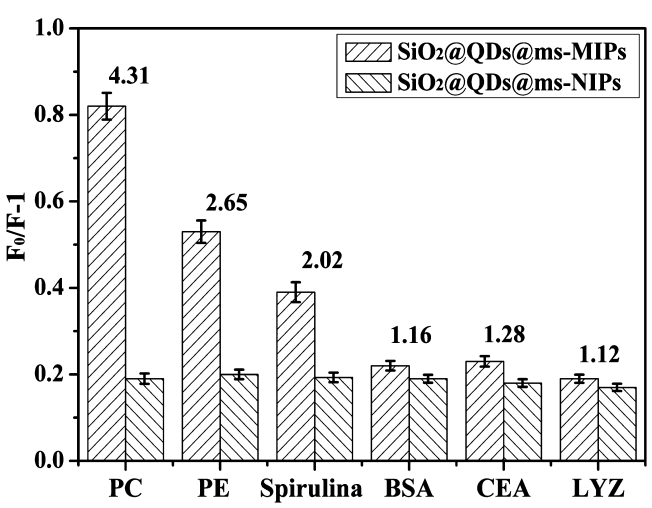

Figure 6. Fluorescent quenching amounts of the $\mathrm{SiO}_{2} @ \mathrm{QDD} @ m s-$ MIPs and $\mathrm{SiO}_{2} @$ QDs@ms-NIPs in the different proteins (phycocyanin (PC), phycoerythrin (PE), spirulina, BSA, CEA, and LYZ) solutions at the same concentration.

fluorescence quenching amount of the MIPs was the highest for phycocyanin, followed by phycoerythrin and spirulina powder, much larger than BSA, CEA, and LYZ which caused very close and quite low fluorescence quenching. Phycoerythrin has a 
structure quite similar to phycocyanin, and it is very likely to result in fluorescence quenching. Spirulina powder contains certain amounts of phycocyanin and phycoerythrin, so quenching effect was also observed. Still, the MIPs could be concluded to be selective for phycocyanin. As for BSA, CEA, and LYZ, this phenomenon can be explained from the following two aspects. First, in the synthesis process, a larger number of specific recognition sites complementary with the template molecules in shape, size, and functionality were generated on the MIPs; hence the template phycocyanin could be strongly rebound to the MIPs and then produce significant fluorescence quenching. However, BSA, CEA, and LYZ with different shapes and molecular weights from phycocyanin were not complementary to the recognition sites and thereby had less chance to quench the QDs fluorescence, although they also could interact with APTES by hydrogen bonding. Second, with fewer carboxyl groups in BSA, CEA, and LYZ, ${ }^{36,37}$ the electrondonating ability of the molecules were weaker, and then the electron-transfer interaction between them with QDs was much weaker. As a result, the fluorescence quenching ability of the possibly interfering proteins was much lower than that of phycocyanin. Therefore, the MIPs probe possessed high selectivity toward the template phycocyanin.

3.7. Practical Application of the Probe to Environmental Water Samples. The practicability of the probe was further evaluated by selective separation/enrichment and sensitive determination of phycocyanin in seawater and lake water samples. First, SDS-PAGE was utilized to visualize protein samples in the biological fluid prepared by seawater. SI Figure S7 shows the SDS-PAGE results. As seen from lane 2, the presented three main bands indicated the existence of phycocyanin, CEA, and BSA according to their molecular weights. Lane 3 shows the supernatant after treatment with the MIPs, in which the band of phycocyanin disappeared obviously and the other two remaining bands rarely changed. Namely, most phycocyanin was specifically captured by the MIPs, while CEA and BSA were not. However, after treatment by NIPs, there were still three main bands found in lane 4, suggesting the NIPs had no selectivity for phycocyanin. On the other hand, quantification of the protein bands was carried out with gray level determination using software BandScan 5.0. The following information could be attained: in lane 2, without treatment, the present amount of phycocyanin was calculated as $27.9 \%$ of the total proteins; in lane 3 , after treatment by MIPs, phycocyanin decreased to $10.7 \%$, which could be roughly estimated that $38.4 \%$ (the ratio of $10.7 \%$ to $27.9 \%$ ) phycocyanin remained and $61.6 \%$ phycocyanin was adsorbed by MIPs. These results indicated that specific recognition sites were generated in the MIPs and the MIPs were practically applicable for selective separation and enrichment of phycocyanin from protein mixtures.

Furthermore, the detection of phycocyanin in seawater and lake water samples was performed to evaluate the practical applicability of the MIPs probe. As listed in Table 1, satisfactory recoveries and accuracies were attained in a range of 95.0$103.5 \%$ with RSDs of $3.2-5.3 \%$ for the spiked seawater samples with three concentrations. As well as, the recoveries for the spiked lake water samples were $94.0-105.0 \%$ with RSDs of $3.6-4.5 \%$. Overall, these results confirmed the developed fluorescent probe was practically feasible for accurate determination of trace phycocyanin in complex environmental water samples.
Table 1. Spiked Recoveries and Relative Standard Deviations (RSDs, \%; $n=3$ ) for the Determination of the Phycocyanin in Seawater and Lake Water Samples Using the $\mathrm{SiO}_{2} @$ QDs@ms-MIPs

\begin{tabular}{cccr} 
sample & spiked $(\mu \mathrm{mol} / \mathrm{L})$ & found $(\mu \mathrm{mol} / \mathrm{L})$ & recovery $\pm \mathrm{RSD}(\%)$ \\
seawater & 0 & 0 & \\
& 0.1 & 0.095 & $95.0 \pm 5.3$ \\
& 0.2 & 0.207 & $103.5 \pm 4.7$ \\
lake water & 0.5 & 0.476 & $95.2 \pm 3.2$ \\
& 0 & 0 & \\
& 0.1 & 0.105 & $105.0 \pm 4.5$ \\
& 0.2 & 0.188 & $94.0 \pm 4.7$ \\
& 0.5 & 0.523 & $104.6 \pm 3.6$ \\
\hline
\end{tabular}

3.8. Method Performance Comparison. The performance of this developed $\mathrm{SiO}_{2} @ Q D s @ m s-M I P s$ method was compared with that of some reported MIPs based QDs methods, as listed in Table 2. ${ }^{17,20-24,38}$ As seen from the table, the reported $\mathrm{CdSe} / \mathrm{ZnS}$ QDs-MIP can qualitatively identify caffeine, but the quantitative determination is quite difficult. ${ }^{38}$ Quantitative analysis has been increasingly reported, for example, MIP-capped CdTe QDs for 2,4,6-trinitrotoluene, ${ }^{20}$ MIP-coated CdTe QDs for cytochrome $c,{ }^{17}$ and epitope MIPcoated CdTe QDs for BSA ${ }^{23}$ but with low sensitivity (Table 2). The low sensitivity problem commonly exists in MIPs based QDs systems. During the preparation process, the MIPs layers are synthesized by encapsulating QDs into highly cross-linked polymers, and QDs are far from the surface of particles. Consequently, the amounts of interaction sites between the target molecule and the functional monomer are relatively low, which reduces the sensitivity of the system. More efforts still need to be made to further enhance the sensitivity of MIPs based QDs systems while retaining their high selectivity. On the other hand, the developed MIP-coated CdSe QDs for ractopamine $^{21}$ and $\mathrm{ZnS} / \mathrm{Mn}$ QDs@MIP for diazinon ${ }^{24}$ attain relatively high sensitivity, but their response time is relatively long (Table 2). Possibly, reducing the MIP-shell thickness and providing (meso)porous structures are two effective ways to improve sensitivity and shorten response time. Excitingly, our developed $\mathrm{SiO}_{2} @ Q D s @ m s-M I P s$ probe system has demonstrated high sensitivity and rapid response for the fluorescent detection of protein. In our present study, the MIPs layers are anchored on the surface of CdTe QDs via a surface imprinting process. The ultrathin imprinting shell layer of about $5 \mathrm{~nm}$ facilitates higher sensitivity and shorter analysis time. Furthermore, the mesoporous structures also play an important role in sensitivity improvement and response rapidity.

\section{CONCLUSIONS}

In summary, a novel mesoporous structured $\mathrm{SiO}_{2} @ \mathrm{QDs} @ m s-$ MIPs probe was fabricated through a sol-gel core-shell imprinting process for the determination of trace phycocyanin based on electron-transfer-induced fluorescence quenching. By combining the strong fluorescence QDs and high selectivity MIPs, the $\mathrm{SiO}_{2} @ Q D s @ m s-M I P s$ demonstrated specific recognition and sensitive detection ability for phycocyanin. The responsiveness, reproducibility, stability, and practicability of the phycocyanin probe were all proved satisfactory. The probe can be utilized as an alternative analytical tool for phycocyanin, which may be greatly favorable for the protein evaluation of physiological, biomedical, and environmental issues. More endeavors should be continuously made to push forward the 
Table 2. Performance Comparison with Other Reported MIP Based QDs Methods

\begin{tabular}{|c|c|c|c|c|c|c|c|}
\hline system & template & mechanism & $\begin{array}{l}\text { response } \\
\text { time }(\mathrm{min})\end{array}$ & linear range & $\begin{array}{l}\text { detection } \\
\text { limit }\end{array}$ & real sample & ref \\
\hline CdSe/ZnS QDs@MIP & caffeine & $\begin{array}{l}\text { QD } \\
\text { photoionization }\end{array}$ & & & & & 38 \\
\hline ZnS/Mn QDs@MIP & diazinon & energy transfer & 120 & $50-600 \mathrm{ng} / \mathrm{mL}$ & $38.6 \mathrm{ng} / \mathrm{mL}$ & tap water & 24 \\
\hline MIP-capped CdTe QDs & 2,4,6-trinitrotoluene & electron transfer & 10 & $0.8-30 \mu \mathrm{M}$ & $0.28 \mu \mathrm{M}$ & soil & 20 \\
\hline MIP-coated CdSe QDs & ractopamine & charge transfer & 40 & $0.00121-3.03 \mu \mathrm{M}$ & $0.76 \mathrm{nM}$ & pork & 21 \\
\hline Si-NP/CdTe/MIP & bovine hemoglobin & electron transfer & & $0.02-2.1 \mu \mathrm{M}$ & $9.4 \mathrm{nM}$ & bovine blood & 22 \\
\hline MIP-coated CdTe QDs & cytochrome $c$ & electron transfer & & $0.97-24 \mu \mathrm{M}$ & $0.41 \mu \mathrm{M}$ & & 17 \\
\hline EMIP $^{a}$-coated CdTe QDs & BSA & electron transfer & & $0.5-10 \mu \mathrm{M}$ & $0.11 \mu \mathrm{M}$ & $\begin{array}{l}\text { bovine blood and } \\
\text { bovine calf serum }\end{array}$ & 23 \\
\hline SiO ${ }_{2} @ \mathrm{CdTe}$ QDs@ms-MIP & phycocyanin & electron transfer & 8 & $0.02-2.4 \mu \mathrm{M}$ & $5.9 \mathrm{nM}$ & seawater & $\begin{array}{l}\text { this } \\
\text { work }\end{array}$ \\
\hline
\end{tabular}

development of QDs@ms-MIPs based probes with better performances. Moreover, the fabrication of MIPs related probes requires integrating additional functional elements. With the rapid advance of various signal units (e.g., QDs) and versatile MIPs, we believe their synergistic effects can provide bright perspectives to develop such composite materials for potential utilizations.

\section{ASSOCIATED CONTENT}

\section{S Supporting Information}

$\mathrm{N}_{2}$ adsorption-desorption isotherms of $\mathrm{SiO}_{2} @ \mathrm{QDs} @ m s-$ MIPs, FT-IR spectra of $\mathrm{SiO}_{2}, \mathrm{SiO}_{2} @ Q D s$, and $\mathrm{SiO}_{2} @ \mathrm{QDs} @$ ms-MIPs, TG and DTG curves of $\mathrm{SiO}_{2} @ Q D s @ m s-M I P s$, adsorption capacity of $\mathrm{SiO}_{2} @ \mathrm{QDs} @ m s-\mathrm{MIPs}$ and $\mathrm{SiO}_{2} @$ QDs@ms-NIPs, fluorescence spectra for the interaction of APTES with phycocyanin, fluorescent spectra of $\mathrm{SiO}_{2} @ \mathrm{QDs} @$ ms-MIPs, SiO $2 @ Q D s @ m s-M I P s$ with phycocyanin, and $\mathrm{SiO}_{2} @$ QDs@ms-NIPs, and SDS-PAGE analysis of spiked protein mixture treated with $\mathrm{SiO}_{2} @ \mathrm{QDs} @ m s-\mathrm{MIPs}$ and $\mathrm{SiO}_{2} @ \mathrm{QDs} @$ ms-NIPs. This material is available free of charge via the Internet at http://pubs.acs.org.

\section{AUTHOR INFORMATION}

\section{Corresponding Author}

*E-mail: 1xchen@yic.ac.cn. Tel./Fax: +86-535 2109130.

\section{Notes}

The authors declare no competing financial interest.

\section{ACKNOWLEDGMENTS}

This work was financially supported by the National Natural Science Foundation of China (Grants 21275158, 21477160, and 21175084), the Scientific Research Foundation for the Returned Overseas Chinese Scholars, State Education Ministry, and the Innovation Projects of the Chinese Academy of Sciences (Grant KZCX2-EW-206).

\section{REFERENCES}

(1) Wilson, R.; Cossins, A. R.; Spiller, D. G. Encoded Microcarriers for High-Throughput Multiplexed Detection. Angew. Chem., Int. Ed. 2006, 45, 6104-6117.

(2) Yu, F.; Li, P.; Wang, B.; Han, K. Reversible Near-Infrared Fluorescent Probe Introducing Tellurium to Mimetic Glutathione Peroxidase for Monitoring the Redox Cycles Between Peroxynitrite and Glutathione in Vivo. J. Am. Chem. Soc. 2013, 135, 7674-7680.

(3) Bustos, A. R. M.; Alfonso, L. T.; Encinar, J. R.; Fernández, J. M. C.; Pereiro, R.; Medel, S. Elemental and Molecular Detection for
Quantum Dots-Based Immunoassays: A Critical Appraisal. Biosens. Bioelectron. 2012, 33, 165-171.

(4) Chen, X.; Zhou, Y.; Peng, X.; Yoon, J. Fluorescent and Colorimetric Probes for Detection of Thiols. Chem. Soc. Rev. 2010, 39, 2120-2135.

(5) Zhu, L.; Cui, X.; Wu, J.; Wang, Z.; Wang, P.; Hou, Y.; Yang, M. Fluorescence Immunoassay Based on Carbon Dots as Labels for the Detection of Human Immunoglobulin G. Anal. Methods 2014, 6, 4430-4436.

(6) Ying, E.; Li, D.; Guo, S.; Dong, S.; Wang, J. Synthesis and BioImaging Application of Highly Luminescent Mercaptosuccinic AcidCoated CdTe Nanocrystals. PloS One 2008, 3, No. e2222.

(7) Chen, H.; Lin, L.; Lin, Z.; Guo, G.; Lin, J. Chemiluminescence Arising from the Decomposition of Peroxymonocarbonate and Enhanced by CdTe Quantum Dots. J. Phys. Chem. A 2010, 114, 10049-10058.

(8) Wang, X.; Sheng, P.; Zhou, L.; Tong, X.; Shi, L.; Cai, Q. Fluorescence Immunoassay of Octachlorostyrene Based on Förster Resonance Energy Transfer Between CdTe Quantum Dots and Rhodamine B. Biosens. Bioelectron. 2014, 60, 52-56.

(9) Shang, L.; Zhang, L.; Dong, S. Turn-on Fluorescent Cyanide Sensor Based on Copper Ion-Modified CdTe Quantum Dots. Analyst 2009, 134, 107-113.

(10) Wolcott, A.; Gerion, D.; Visconte, M.; Sun, J.; Schwartzberg, A.; Chen, S.; Zhang, J. Silica-Coated CdTe Quantum Dots Functionalized with Thiols for Bioconjugation to IgG Proteins. J. Phys. Chem. B 2006, 110, 5779-5789.

(11) Wu, C.; Zheng, J.; Huang, C.; Lai, J.; Li, S.; Chen, C.; Zhao, Y. Hybrid Silica- Nanocrystal-Organic Dye Superstructures as PostEncoding Fluorescent Probes. Angew. Chem., Int. Ed. 2007, 46, 53935396.

(12) Chen, L.; Xu, S.; Li, J. Recent Advances in Molecular Imprinting Technology: Current Status, Challenges and Highlighted Applications. Chem. Soc. Rev. 2011, 40, 2922-2942.

(13) Whitcombe, M. J.; Chianella, I.; Larcombe, L.; Piletsky, S. A.; Noble, J.; Porter, R.; Horgan, A. The Rational Development of Molecularly Imprinted Polymer-Based Sensors for Protein Detection. Chem. Soc. Rev. 2011, 40, 1547-1571.

(14) Li, J.; Zhang, Z.; Xu, S.; Chen, L. Label-Free Colorimetric Detection of Trace Cholesterol Based on Molecularly Imprinted Photonic Hydrogels. J. Mater. Chem. 2011, 21, 19267-19274.

(15) Lu, C. H.; Zhou, W. H.; Han, B.; Yang, H.; Chen, X.; Wang, X. Surface-Imprinted Core-Shell Nanoparticles for Sorbent Assays. Anal. Chem. 2007, 79, 5457-5461.

(16) Zhang, Z.; Chen, L.; Yang, F.; Li, J. Uniform Core-Shell Molecularly Imprinted Polymers: A Correlation Study Between Shell Thickness and Binding Capacity. RSC Adv. 2014, 4, 31507-31514.

(17) Zhang, W.; He, X. W.; Chen, Y.; Li, W.; Zhang, Y. Composite of CdTe Quantum Dots and Molecularly Imprinted Polymer as a Sensing Material for Cytochrome. Biosens. Bioelectron. 2011, 26, 2553-2558.

(18) Zhang, W.; He, X.; Li, W.; Zhang, Y. Thermo-Sensitive Imprinted Polymer Coating CdTe Quantum Dots for Target Protein 
Specific Recognition. Chem. Commun. (Cambridge, U. K.) 2012, 48, 1757-1759.

(19) Ge, S.; Lu, J.; Ge, L.; Yan, M.; Yu, J. Development of a Novel Deltamethrin Sensor Based on Molecularly Imprinted Silica Nanospheres Embedded CdTe Quantum Dots. Spectrochim. Acta, Part A 2011, 79, 1704-1709.

(20) Xu, S.; Lu, H.; Li, J.; Song, X.; Wang, A.; Chen, L.; Han, S. Dummy Molecularly Imprinted Polymers-Capped CdTe Quantum Dots for the Fluorescent Sensing of 2,4,6-Trinitrotoluene. ACS Appl. Mater. Interfaces 2013, 5, 8146-8154.

(21) Liu, H.; Fang, G.; Wang, S. Molecularly Imprinted Optosensing Material Based on Hydrophobic CdSe Quantum Dots via a Reverse Microemulsion for Specific Recognition of Ractopamine. Biosens. Bioelectron. 2014, 55, 127-132.

(22) Li, D.; He, X.; Chen, Y.; Li, W.; Zhang, Y. Novel Hybrid Structure Silica/CdTe/Molecularly Imprinted Polymer: Synthesis, Specific Recognition, and Quantitative Fluorescence Detection of Bovine Hemoglobin. ACS Appl. Mater. Interfaces 2013, 5, 1260912616.

(23) Yang, Y. Q.; He, X. W.; Wang, Y.; Li, W.; Zhang, Y. Epitope Imprinted Polymer Coating CdTe Quantum Dots for Specific Recognition and Direct Fluorescent Quantification of the Target Protein Bovine Serum Albumin. Biosens. Bioelectron. 2014, 54, 266272.

(24) Zhao, Y.; Ma, Y.; Li, H.; Wang, L. Composite QDs@MIP Nanospheres for Specific Recognition and Direct Fluorescent Quantification of Pesticides in Aqueous Media. Anal. Chem. 2011, 84, 386-395.

(25) Lofgreen, J. E.; Ozin, G. A. Controlling Morphology and Porosity to Improve Performance of Molecularly Imprinted Sol-Gel Silica. Chem. Soc. Rev. 2014, 43, 911-933.

(26) Marx, S.; Zaltsman, A.; Turyan, I.; Mandler, D. Parathion Sensor Based on Molecularly Imprinted Sol-Gel Films. Anal. Chem. 2004, 76, $120-126$.

(27) MunáJung, B.; SooáKim, M.; JináKim, W.; YoungáChang, J. Molecularly Imprinted Mesoporous Silica Particles Showing a Rapid Kinetic Binding. Chem. Commun. (Cambridge, U. K.) 2010, 46, 36993701.

(28) Kang, C.; Li, W.; Tan, L.; Li, H.; Wei, C.; Tang, Y. Highly Ordered Metal Ion Imprinted Mesoporous Silica Particles Exhibiting Specific Recognition and Fast Adsorption Kinetics. J. Mater. Chem. A 2013, 24, 7147-7153.

(29) Eriksen, N. T. Production of Phycocyanin-A Pigment with Applications in Biology, Biotechnology, Foods and Medicine. Appl. Microbiol. Biotechnol. 2008, 80, 1-14.

(30) Song, K.; Li, L.; Li, Z.; Tedesco, L.; Hall, B.; Shi, K. Remote Detection of Cyanobacteria through Phycocyaninfor Water Supply Source Using Three-Band Model. Ecol. Inf. 2013, 15, 22-33.

(31) Zhang, Z.; Li, J.; Fu, J.; Chen, L. Fluorescent and Magnetic Dual-Responsive Coreshell Imprinting Microspheres Strategy for Recognition and Detection of Phycocyanin. RSC Adv. 2014, 4, 20677-20685.

(32) Zhang, Z.; Li, J.; Fu, L.; Liu, D.; Chen, L. Magnetic Molecularly Imprinted Microsensors for Selective Recognition and Transport of Fluorescent Phycocyanin in Seawater. J. Mater. Chem. A 2015, DOI: $10.1039 / \mathrm{c} 5$ ta00143a.

(33) Stober, W.; Fink, A. Controlled Growth of Monodisperse Silica Spheres in the Micron Size Range. J. Colloid Interface Sci. 1968, 26, 62-69.

(34) Wang, C.; Ma, Q.; Dou, W.; Kanwal, S.; Wang, G.; Yuan, P.; Su, X. Synthesis of Aqueous CdTe Quantum Dots Embedded Silica Nanoparticles and Their Applications as Fluorescence Probes. Talanta 2009, 77, 1358-1364.

(35) Cheng, P. P. H.; Silvester, D.; Wang, G.; Kalyuzhny, G.; Douglas, A.; Murray, R. W. Dynamic and Static Quenching of Fluorescence by 1-4 nm Diameter Gold Monolayer-Protected Clusters. J. Phys. Chem. B 2006, 110, 4637-4644.

(36) Brejc, K.; Ficner, R.; Huber, R.; Steinbacher, S. Isolation, Crystallization, Crystal Structure Analysis and Refinement of
Allophycocyanin from the Cyanobacterium Spirulina Platensis at 2.3 Å Resolution. J. Mol. Biol. 1995, 249, 424-440.

(37) Yin, G.; Li, C.; He, Q.; Liu, Z.; Yuan, N.; Ding, F. Isolation and Characterization of Biliproteins and Polysaccharides from Spirulina. Fine Chem. 1999, 16, 10-13.

(38) Lin, C. I.; Joseph, A. K.; Chang, C. K.; Lee, Y. D. Synthesis and Photoluminescence Study of Molecularly Imprinted Polymers Appended onto Cdse/Zns Core-Shells. Biosens. Bioelectron. 2004, 20, 127-131. 